\title{
Ultrafast molecular orbital tomography of a pentacene thin film using time-resolved momentum microscopy at a free-electron laser
}

Markus Scholz ( $\square$ markus.scholz@xfel.eu )

European X-Ray Free-Electron Laser Facility

Kiana Baumgärtner

Julius-Maximilians-Universität

Christian Metzger

Julius-Maximilians-Universität

Dmytro Kutnyakhov

DESY Photon Science https://orcid.org/0000-0002-6294-4600

Michael Heber

Deutsches Elektronen-Synchrotron DESY

\section{Federico Pressacco}

Deutsches Elektronen-Synchrotron DESY https://orcid.org/0000-0002-8299-4269

Chul-Hee Min

Institut für Experimentelle und Angewandte Physik

Thiago Peixoto

Universität Würzburg https://orcid.org/0000-0001-6629-736X

Mario Reiser

University of Siegen

Chan Kim

European X-Ray Free-Electron Laser Facility

Wei Lu

European X-Ray Free-Electron Laser Facility

Roman Shayduk

European X-Ray Free-Electron Laser Facility

Manuel Izquierdo

European X-Ray Free-Electron Laser Facility

Günter Brenner

Deutsches Elektronen-Synchrotron DESY, Notkestr. 85, D-22607 Hamburg, Germany

Friedrich Roth

TU Bergakademie Freiberg

Achim Schoell 
Julius-Maximilians-Universität

Serguei Molodtsov

European X-Ray Free Electron Laser (XFEL)

Wilfried Wurth

Universität Hamburg

Friedrich Reinert

Würzburg University

Anders Madsen

European XFEL https://orcid.org/0000-0001-6594-1029

\section{Article}

Keywords:

Posted Date: July 27th, 2020

DOI: https://doi.org/10.21203/rs.3.rs-40911/v1

License: (c) (i) This work is licensed under a Creative Commons Attribution 4.0 International License. Read Full License

Version of Record: A version of this preprint was published at Nature Communications on May 18th, 2022. See the published version at https://doi.org/10.1038/s41467-022-30404-6. 


\title{
Ultrafast molecular orbital tomography of a pentacene thin film using time-resolved momentum microscopy at a free-electron laser
}

\author{
M. Scholz ${ }^{1, *}$, K. Baumgärtner ${ }^{2}$, C. Metzger ${ }^{2}$, D. Kutnyakhov ${ }^{3}$, M. Heber ${ }^{3}$, F. Pressacco ${ }^{3}$, \\ C.H. Min ${ }^{2,4}$, T.R.F. Peixoto ${ }^{2}$, M. Reiser ${ }^{1}$, C. Kim ${ }^{1}$, W. Lu ${ }^{1}$, R. Shayduk ${ }^{1}$, W. M. Izquierdo ${ }^{1}$, G. \\ Brenner $^{3}$, F. Roth ${ }^{5}$, A. Schöll ${ }^{2}$, S. Molodtsov ${ }^{1,5}$, W. Wurth $\dagger^{3,6,7}$, F. Reinert ${ }^{2}$, and \\ A. Madsen ${ }^{1}$ \\ ${ }^{1}$ European X-Ray Free-Electron Laser Facility, Holzkoppel 4, 22869 Schenefeld, Germany \\ ${ }^{2}$ Experimentelle Physik 7, Julius-Maximilians-Universität, Am Hubland, 97074 Würzburg, Germany \\ ${ }^{3}$ Deutsches Elektronen-Synchrotron DESY, Notkestrasse 85, 22607 Hamburg, Germany \\ ${ }^{4}$ Institut für Experimentelle und Angewandte Physik, Christian-Albrechts-Universität zu Kiel, 24098 Kiel, Germany \\ ${ }^{5}$ Institute of Experimental Physics, TU Bergakademie Freiberg, Leipziger Strasse 23, 09599 Freiberg, Germany \\ ${ }^{6}$ The Hamburg Centre for Ultrafast Imaging (CUI), Luruper Chaussee 149, 22761 Hamburg, Germany \\ ${ }^{7}$ Department Physik and Centre for Free-Electron Laser Science, Universität Hamburg, Luruper Chaussee 149, \\ 22761 Hamburg, Germany \\ *To whom correspondence should be addressed; markus.scholz@xfel.eu.
}

\begin{abstract}
Understanding and control of photon-induced dynamics of molecules on solid surfaces, including atomic rearrangements as well as charge transfer and non-equilibrium electron dynamics, are of essential importance for surface chemistry but also for the development of new devices. We use time-resolved momentum microscopy at a free-electron laser (FEL) and extend orbital tomography to time-resolved imaging of electronic wave functions of excited molecular orbitals. This technique will provide unprecedented insight into the ultrafast interplay between structural and electronic dynamics. In this work we prove general applicability and establish the experimental conditions at FEL sources to minimize space charge effects and radiation damage. We investigate a bilayer pentacene film on $\mathrm{Ag}(110)$ by optical laser pump and FEL probe experiments. From the momentum microscopy signal, we obtain time-dependent momentum maps of the molecular valence states that can be related to the molecular initial states by simulations of the involved photoemission matrix elements. A state above the Fermi level is identified which is temporarily occupied after optical excitation.
\end{abstract}

\section{Introduction}

Photon-induced phenomena of adsorbates on solid surfaces have been intensively investigated over the last decade ${ }^{1-4}$. Despite some success in controlling physical properties and initiating femtochemistry by light, the description of ultrafast dynamics upon excitation remains challenging. Significant advances in photo-induced surface chemistry require deciphering the complex interplay between excited electronic wave packet dynamics as well as the rapid rearrangement of atomic positions, and interactions at the metal-organic interface. Angle-resolved photoelectron spectroscopy (ARPES) is a well-known and powerful method to investigate the electronic structure of molecules. In the last decade, orbital tomography has emerged as an exciting extension of the photoemission technique for imaging localized electronic wave functions in thin film molecules ${ }^{5-9}$. In this framework, the photoemission process can be described either in a one-step model where the final state is represented by a plane wave or using more sophisticated final state approximations ${ }^{10-13}$. Although the phase of the electronic wave function is not an observable, it can be retrieved under suitable experimental conditions ${ }^{14}$, or by use of iterative algorithms traditionally employed in coherent diffraction imaging ${ }^{15,16}$. This enables more intricate data analyses, like reconstructing of the electron density of molecular orbitals in real space.

Expanding the orbital tomography technique into the time domain requires XUV or x-ray photon energies and ultrashort pulses with sufficient flux presently only provided by self-amplified spontaneous emission (SASE) free-electron lasers (FELs) or high harmonics generation (HHG) sources. Most available HHG systems are operating at rather low repetition rates $(\sim 1 \mathrm{kHz})$ at $1 \mu \mathrm{J}$ pulse energy ${ }^{17}$ and are therefore very time consuming for conducting pump-probe experiments. FELs based 
on superconducting linear accelerators such as FLASH at DESY in Hamburg ${ }^{18}$ or the European XFEL with repetition rates ranging from hundreds of $\mathrm{kHz}$ to $\mathrm{MHz}$ are thus ideal sources for time-resolved orbital tomography. However, SASE pulses have strong shot-to-shot intensity fluctuation and the data must be sorted by temporal jitter and pulse energy accordingly. The high brightness of a FEL can lead to radiation-induced damage in the sample and unwanted space charge effects in photoelectron experiments that limit the energy and momentum resolution in time- and angle-resolved photoemission spectroscopy ${ }^{19}$. For soft matter, in particular, the influence of radiation damage may result in significant peak broadening or side features in the spectra caused by bond-breaking or radical formation ${ }^{20}$. A careful survey of the sample degradation under illumination at various light intensities and time scales is therefore crucial to gauge and suppress radiation damage. The effects of optical pump and $\mathrm{x}$-ray probe pulse-induced space charge in photoemission were intensely studied over the last years, both from a theoretical point of view and by experiments ${ }^{19,21-23}$. Coulomb interaction between excited photoelectrons during and after the FEL pulse can lead to a significant shift in binding energy, spectral broadening, and a smeared out photoelectron angular distribution. Pump laser multiphoton excitation results in a cloud of slow electrons in the vicinity of the sample surface and perpendicular to the axis of the flight path in the time-of-flight (TOF) instrument. On their trajectories towards the detector they can interact with faster photoelectrons passing by and cause an additional shift and broadening in the detected photoemission signal. It is thus essential to reduce the number of slow electrons and photoelectrons per bunch by attenuating the optical laser and FEL ${ }^{19,21}$.

Pentacene is a prominent example for complex exciton dynamics and singlet fission and has attracted considerable interest due to its potential of exceeding the Shockley-Queisser limit in solar energy conversion ${ }^{24}$. Theoretical and experimental investigations focus on how the optically excited exciton is redistributed across multiple molecules as well as the convertion into low-energy electron-hole pairs on ultrafast time scales. It is believed that the dynamics of these phenomena are guided by a complex interplay between excited state configurations and charge transfer states. For pentacene films rapid internal conversion of photoexcited states into dark states of multi-exciton character and splitting into two triplets has been reported ${ }^{25}$. Studying the formation and relaxation dynamics of charge carriers and electron transfer between molecules and at the molecule-substrate interface are of crucial importance for the optimization of organic device properties.

Specifically, we investigate a bilayer of pentacene atop $\operatorname{Ag}(110)$. This system exhibits a well-ordered growth structure with high reproducibility. As recently shown ${ }^{11}$, the spectral features in the electronic structure of the first and second layer are well separated in energy from each other. While in the bottom layer the lowest unoccupied molecular orbital (LUMO) is partly filled due to charge transfer from the substrate, the top layer is largely electronically decoupled. This simplifies the identification and reconstruction of the orbitals of individual layers and the capturing of ultrafast charge transfer dynamics within frontier orbitals and at the molecule-substrate interface on a femtosecond time scale.

\section{Method}

The experiments were performed at the PG2 beamline at $\mathrm{FLASH}^{26}$. The experimental geometry is depicted in Fig. 1 (a). Pump and probe pulses impinge on the sample at a polar angle of $\theta=68^{\circ}$ and an azimuthal angle of $\phi=64^{\circ}$ with respect to the [-1 10$]$ direction of the $\operatorname{Ag}(110)$ surface, and are aligned to have spacial overlap. The photoelectrons emitted into the hemisphere above the sample are detected by a novel TOF momentum microscope ${ }^{27,28}$ and recorded according to their wave vector and kinetic energy. The photon wavelength of the FEL was $35 \mathrm{~nm}$ and the average FEL pulse energy of $30 \mu \mathrm{J}$ was attenuated by nitrogen gas and thin film filter foils to acquire spectra similar to reference measurements in static experiments ${ }^{11}$. The energy resolution in the experiment was $80 \mathrm{meV}$ (see supplement, Fig. 1 (b)). The optical pump laser provides a maximum flux of $1 \mathrm{~mJ} / \mathrm{cm}^{2}$ at $400 \mathrm{~nm}$ wavelength, and is synchronized with the FEL to allow for pump-probe delay scans. Within a so-called bunch train of 330 pulses with $1 \mu$ s spacing, at a repetition rate of $10 \mathrm{~Hz}, 299$ pulses are optically pumped, while the remaining 31 are unpumped. The temporal overlap of FEL and optical laser $\left(\tau_{\text {Delay }}=0\right)$ is established by analyzing the intensity evolution at the Fermi edge of a bilayer of pentacene on $\mathrm{Ag}(110)$. The beam spot size of the FEL and optical laser at the sample position is about $250 \mu \mathrm{m} \times 150 \mu \mathrm{m}$ and $260 \mu \mathrm{m} \times 150 \mu \mathrm{m}$, respectively. For capturing the dynamics, the pump pulses were synchronized with the FEL in such a way that the optical delay can be chosen freely between several tens of femtoseconds to tens of picoseconds with respect to the FEL bunch. Pentacene (purity $99 \%$, purchased from Sigma-Aldrich) was deposited from a homemade Knudsen cell evaporator at a deposition rate of one monolayer per $30 \mathrm{~min}$. Before conducting the photoemission experiment the film thickness and quality were verified by low-energy electron diffraction (LEED), evidencing the pentacene bilayer structure on $\operatorname{Ag}(110)^{11}$ (see supplement, Fig. 1 (a)). In this superstructure, all molecules are aligned with the long molecular axis along the [001] direction of the silver substrate. The sample was kept at room temperature during the deposition and experiment. 
(a)

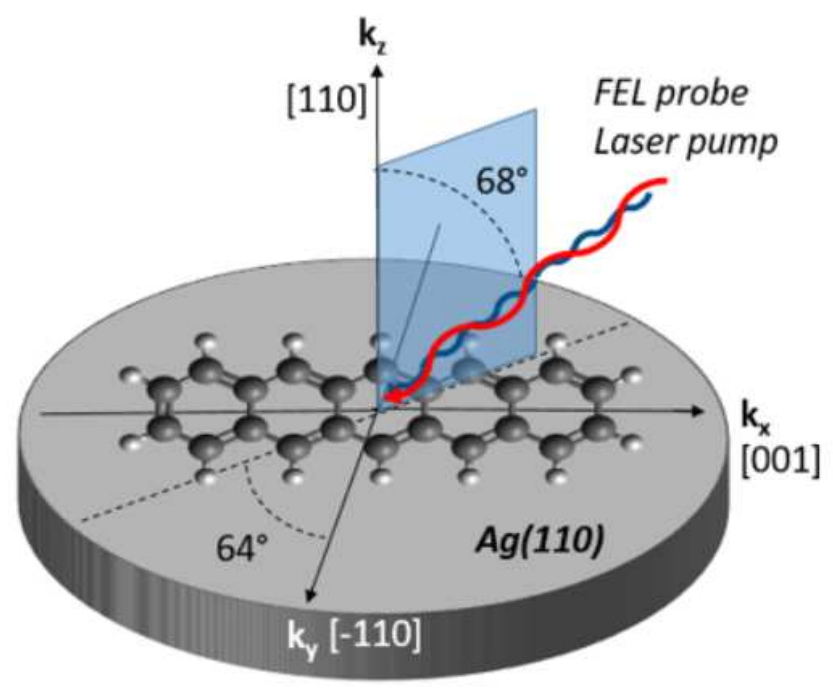

(b)

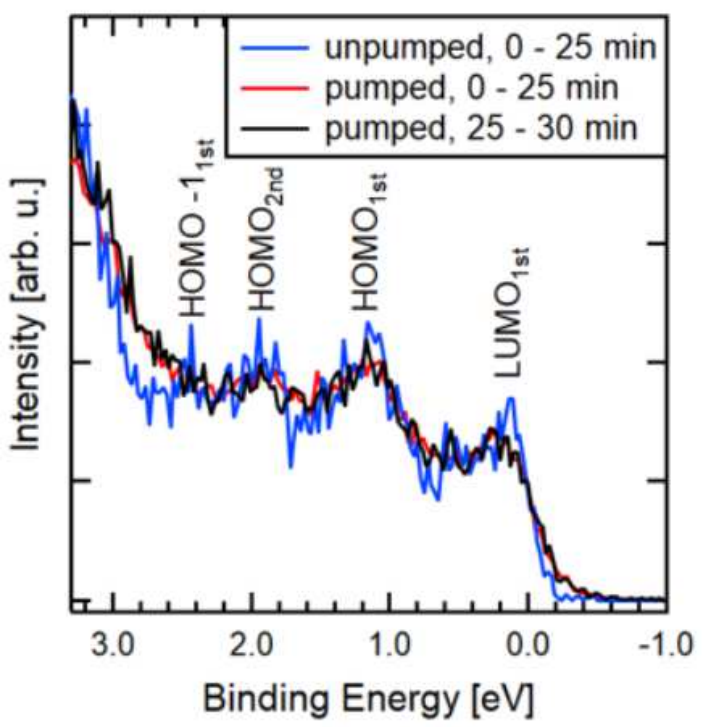

Figure 1. (a) Schematic illustration of the experimental geometry. All of the pentacene molecules are adsorbed with the long molecular axis along the [0 01 1] direction of the silver substrate. Pump and probe pulses are aligned to coincide in the same sample region. (b) Time-integrated photoelectron spectra for a bilayer of pentacene on $\mathrm{Ag}(110)$ with an excitation energy of $\mathrm{h} v=35 \mathrm{eV}$. The measurements are taken at the same sample position for a total measurement time of $30 \mathrm{~min}$. Within a bunch train of 330 pulses with $1 \mu$ s spacing at a repetition rate of $10 \mathrm{~Hz}, 299$ bunches are optically pumped (red and black lines). The blue line shows the time integrated signals of the 31 unpumped bunches. $\mathrm{LUMO}_{1 \mathrm{st}}, \mathrm{HOMO}_{1 \mathrm{st}}, \mathrm{HOMO}-1_{1 \mathrm{st}}$ of the first layer and $\mathrm{HOMO}_{2 \mathrm{nd}}$ of the second layer are indicated in the spectra. The spectra are normalized at $2.2 \mathrm{eV}$ binding energy. The $\mathrm{LUMO}_{1 \mathrm{st}}$ is partly filled due to charge transfer from the substrate.

\section{Results and Discussion}

Fig. 1 (b) displays the normalized, angle-integrated energy distribution curve (EDC) of the frontier orbitals measured with $35 \mathrm{eV}$ photon energy, during constant illumination at the same sample position. The red curve shows the pumped photoemission signal integrated over the first $25 \mathrm{~min}$ after the beginning of the illumination together with the following 5 min of measurement (black) and the parallel detection of the unpumped photoemission signal (blue). Several molecular features as well as the onset of the $\mathrm{Ag} 4 \mathrm{~d}$-bands towards higher binding energies are visible in the recorded valence region: the partly filled LUMO of the first layer $\left(\mathrm{LUMO}_{1 \mathrm{st}}\right)$, the highest occupied molecular orbital (HOMO) of the first layer $\left(\mathrm{HOMO}_{1 \mathrm{st}}\right)$, the HOMO of the second layer $\left(\mathrm{HOMO}_{2 \mathrm{nd}}\right)$, and the second highest occupied molecular orbital (HOMO-1) of the first layer (HOMO- $\left.1_{1 \mathrm{st}}\right)$. Even though molecular thin films tend to be susceptible to temperature- and light-induced deterioration ${ }^{20}$, there is no observable radiation damage within the error of the photoemission intensity for a total measurement time of 30 min at the same sample position reasoned by the similarity of the red and black curves in Fig. 1 (b). As expected for the optically pumped photoemission signal, the spectral features are only marginally broadened in energy due to additional space charge-induced effects from slow electrons. They are also shifted in binding energy by about $150 \mathrm{meV}$ with respect to the EDC obtained from the 31 unpumped pulses (Fig. 1 (b), blue). Note, this energy shift is corrected in Fig. 1 (b). Nevertheless, they are still easily discernible in the EDC and can be assigned to their corresponding molecular initial states by comparison to theoretical photoemission calculations.

Fig. 2 (a-d) displays the experimental time-integrated photoelectron momentum maps (PMMs) of the pentacene film. All momentum maps are integrated in an energy window of $500 \mathrm{meV}$ centered at the maximum intensity position of $\mathrm{LUMO}_{1 \mathrm{st}}$, $\mathrm{HOMO}_{1 \mathrm{st}}, \mathrm{HOMO}_{2 \mathrm{nd}}$, and HOMO-1 $1_{1 \mathrm{st}}$. These four states in the valence region can unambiguously be identified as molecular orbitals. Below the Fermi edge, the central feature close to normal emission and present in all experimental PMMs can be attributed to a silver substrate state also visible on clean $\operatorname{Ag}(110)$ as illustrated in Fig. 2 (e). In Fig. 2 (f-i) we present the calculated PMMs of $\mathrm{LUMO}_{1 \mathrm{st}}, \mathrm{HOMO}_{1 \mathrm{st}}, \mathrm{HOMO}_{2 \text { nd }}$, and $\mathrm{HOMO} 1_{1 \mathrm{st}}$. To this end, we performed simulations of the entire photoemission process with Fermi's Golden Rule in a one-step model. The computational implementation relies on a linear combination of atomic orbitals (LCAO) and independent atomic center (IAC) approximations and is similar to methods already 

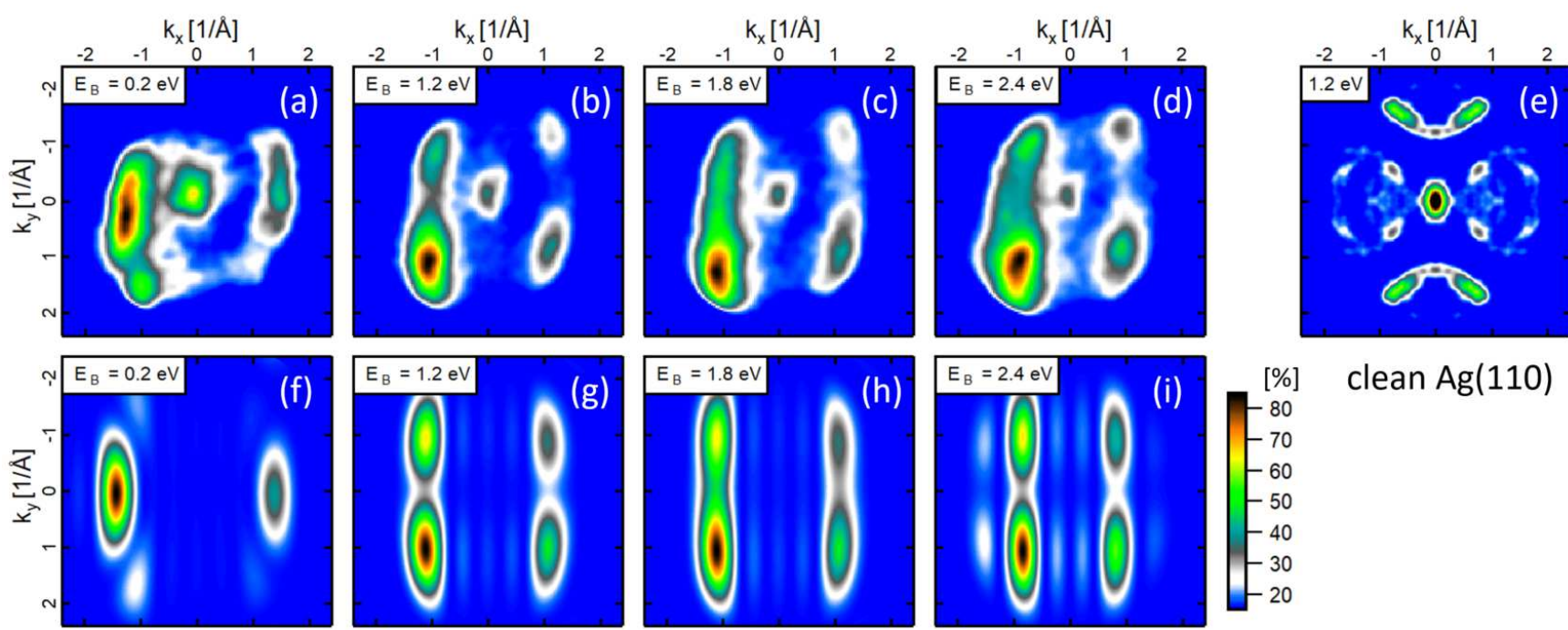

clean $\mathrm{Ag}(110)$

$\mathrm{LUMO}_{1 \mathrm{st}}$

$\mathrm{HOMO}_{1 \mathrm{st}}$

$\mathrm{HOMO}_{2 \mathrm{nd}}$

HOMO-1 $1_{1 \mathrm{st}}$

Figure 2. Time-integrated measured $(\mathrm{a}-\mathrm{d})$ and simulated $(\mathrm{f}-\mathrm{i})$ photoelectron momentum maps (PMMs) for the pentacene valence orbitals at an excitation energy of $\mathrm{h} v=35 \mathrm{eV}$ under simultaneous illumination with the pump and probe pulses. The partly filled $\mathrm{LUMO}_{1 \mathrm{st}}(\mathrm{a})$, the $\mathrm{HOMO}_{1 \mathrm{st}}$ (b), $\mathrm{HOMO}_{2 \text { nd }}$ (c), and the HOMO-1 ${ }_{1 \mathrm{st}}$ (d) can be clearly distinguished from one another and identified by comparison to the simulations (f-i). (e) An unpumped, symmetrized PMM of clean $\operatorname{Ag}(110)$ at $\mathrm{E}_{\mathrm{B}}=1.2 \mathrm{eV}$ with otherwise identical parameters for comparison. The color scale has been adjusted to suppress the background signal.

detailed in literature ${ }^{11,13,29-31}$. The initial state is determined by density functional theory (DFT) calculations of an isolated molecule $^{32}$, while the final state is constructed from a coherent superposition of partial final states at each atomic site. In the simulation we consider the phase shift of the photoelectron induced by the partial wave emitting atom and the inelastic damping inside the molecular film. There is good agreement between the experimental and calculated results of the molecular features, including the spectral weight asymmetry resulting from the experimental geometry and polarization of the probe pulse (see supplement, Fig. 3). While similar comparisons are routine in the evaluation of static photoemission data, they are unprecedented in a pump-probe scheme at a FEL.

Besides these time-integrated data sets, the high repetition rate of the FEL and high efficiency of the momentum microscope enables resolving the time-dependent PMMs down to the femtosecond scale, as depicted in Fig. 3 (a) for the photoemission signal of $\mathrm{LUMO}_{1 \mathrm{st}}, \mathrm{HOMO}_{1 \mathrm{st}}, \mathrm{HOMO}_{2 \mathrm{nd}}$ and at $\mathrm{E}_{\mathrm{B}}=0.2 \mathrm{eV}, \mathrm{E}_{\mathrm{B}}=1.2 \mathrm{eV}$, and $\mathrm{E}_{\mathrm{B}}=1.8 \mathrm{eV}$ binding energy, respectively. The PMMs have been integrated over $350 \mathrm{fs}$ at the indicated pump-probe delay $\tau_{\text {Delay }}$ and slightly smoothed in momentum space with a Gaussian filter. Contour lines A, C and D illustrate the calculated PMMs (see Fig. $2(\mathrm{f}-\mathrm{h})$ ) for the respective molecular orbital. For clarity, we show the calculation only in the left part of the PMM. The data quality permits an unambiguous comparison with theoretical predictions. The intensity around $\Gamma$-point (circle B) is highlighted by a dashed circles. For analyzing the orbital shape, we compare calculated momentum distribution curves (MDCs) with the experimental results (Fig. 3 (b)). The most significant MDCs were taken in the direction as indicated by the dashed black line in the corresponding PMM of Fig. 3 (a) and chosen to highlight relevant features. Note that the calculated MDCs in Fig. 3(b) are for a free molecule and thus without substrate contribution, molecule-molecule or molecule-substrate interaction. Calculations and experimental data show a qualitatively good agreement with the main characteristic features of LUMO and HOMO.

As already apparent for $\tau_{\text {Delay }}<0$ and in Fig. 1 (b), the LUMO is partially occupied due to charge transfer from the substrate. After optical excitation, the characteristic molecular features of $\mathrm{LUMO}_{1 \mathrm{st}}$ and $\mathrm{HOMO}_{1 \mathrm{st}}$ show subtle changes while $\mathrm{HOMO}_{2 \mathrm{nd}}$ remains mainly unaffected. The intensity of the molecular features of $\mathrm{HOMO}_{1 \mathrm{st}}$ drop after time zero while the intensity of the partly occupied $\mathrm{LUMO}_{1 \text { st }}$ slightly increases. The most significant intensity change is around the $\Gamma$-point (Fig. 3, circle B). For both, $\mathrm{LUMO}_{1 \mathrm{st}}$ and $\mathrm{HOMO}_{1 \mathrm{st}}$, the intensity at $\mathrm{B}$ increases after time zero and drops after $\tau_{\text {Delay }}=200 \mathrm{fs}$. The observed dynamics of the wavepacket can be explained in the framework of charge transfer between molecules and substrate ${ }^{33}$. After time zero, charge is redistributed from $\mathrm{HOMO}_{1 \mathrm{st}}$ to the partly filled $\mathrm{LUMO}_{1 \mathrm{st}}$, apparent in Fig. 3 (b) by the variation of the 
(a)
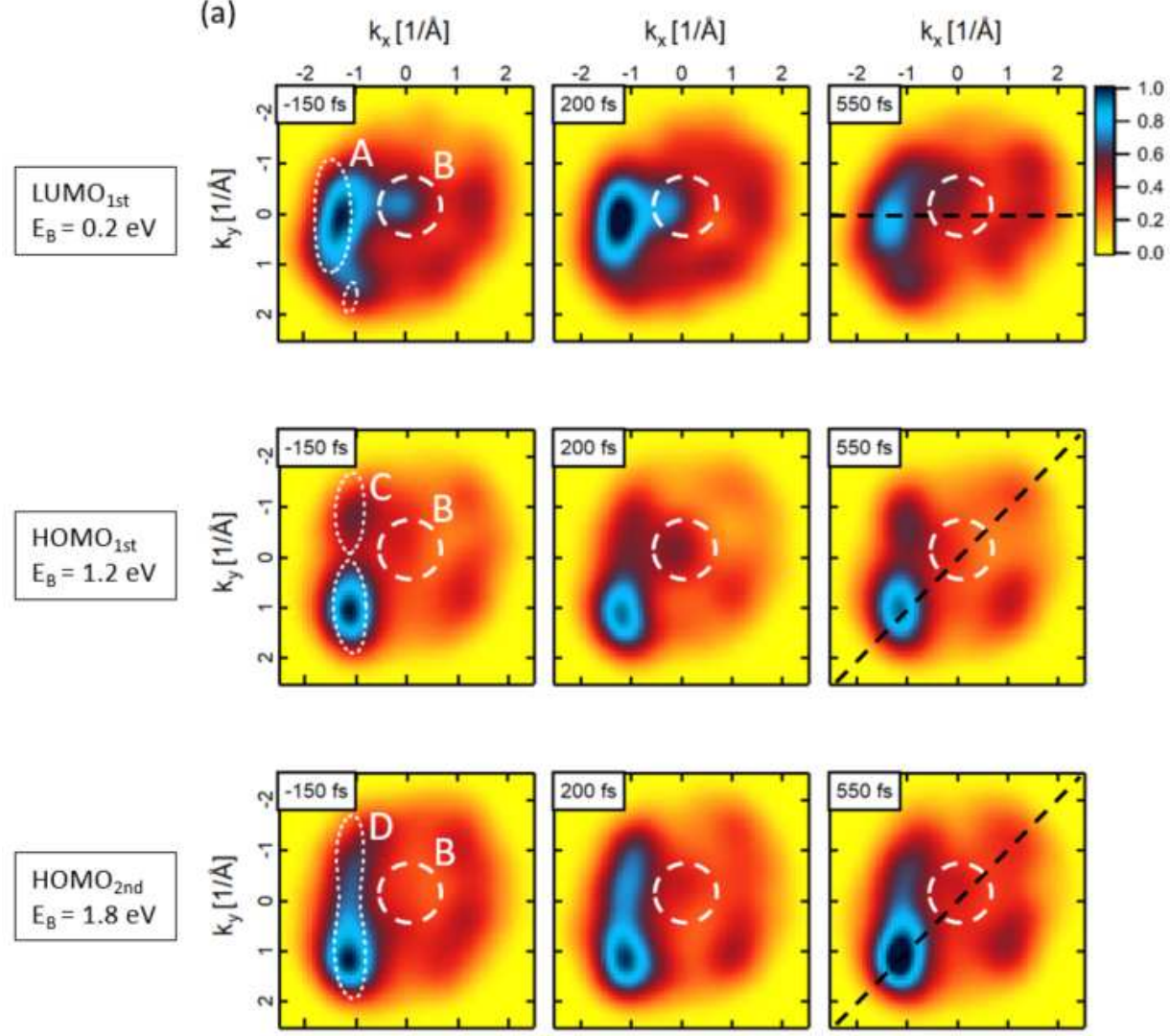

(b)
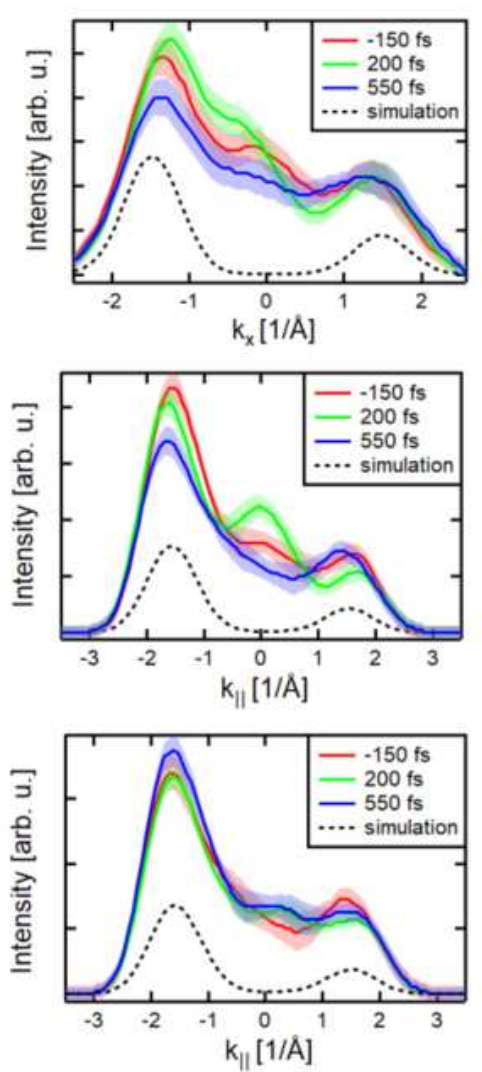

Figure 3. (a) Time evolution of photoelectron momentum maps (PMMs) of pentacene of the $\mathrm{LUMO}_{1 \mathrm{st}}$ (top row), $\mathrm{HOMO}_{1 \mathrm{st}}$ (middle row) and $\mathrm{HOMO}_{2 \mathrm{nd}}$ (bottom row) at $\mathrm{E}_{\mathrm{B}}=0.2 \mathrm{eV}, \mathrm{E}_{\mathrm{B}}=1.2 \mathrm{eV}$ and $\mathrm{E}_{\mathrm{B}}=1.8 \mathrm{eV}$ binding energy, respectively. The PMMs are integrated in intervals of $350 \mathrm{fs}$ for increasing pump-probe delays and smoothed in momentum space with a Gaussian filter. Contour lines A, C, D show the calculated PMMs (see Fig. $2(\mathrm{f}-\mathrm{h})$ ) for the respective molecular orbitals. For clarity, the calculation is only shown on the left side of the PMM. The circle B indicates the intensity around the $\Gamma$-point. (b) Momentum distribution curves for $\mathrm{LUMO}_{1 \mathrm{st}}$ (top), $\mathrm{HOMO}_{1 \mathrm{st}}$ (middle) and $\mathrm{HOMO}_{2 \mathrm{nd}}$ (bottom) in the direction as indicated by the black dashed lines in the corresponding PMMs. The shaded bands reflect 1- $\sigma$ error-bars. 
(a)

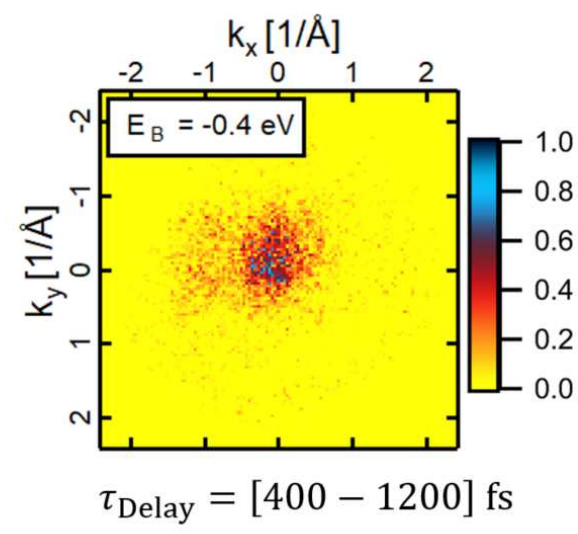

(b)

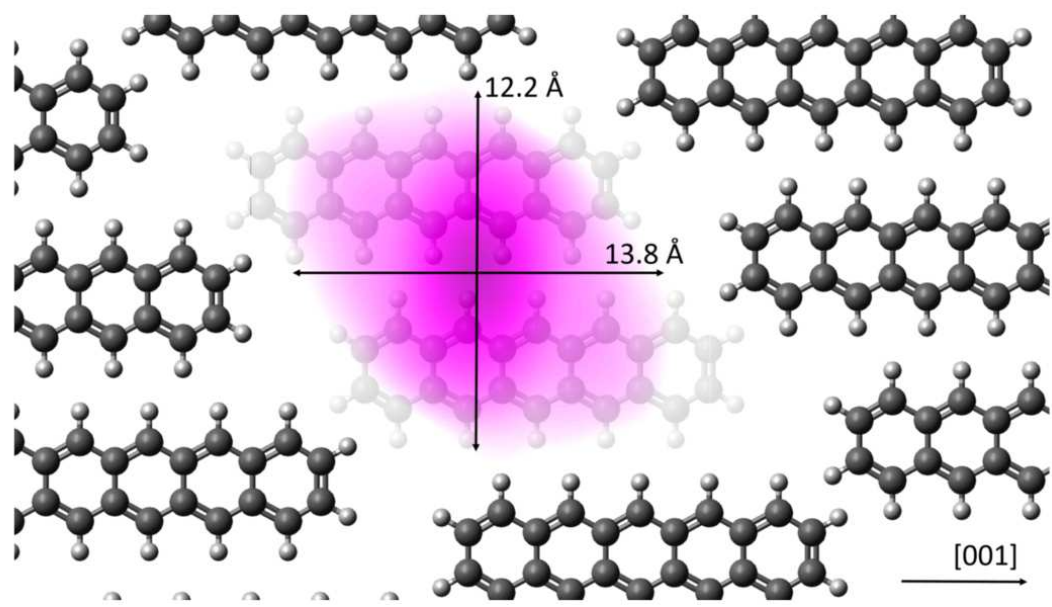

Figure 4. (a) Optical pumping enables the detection of an excited feature at $E_{B}=-0.4 \mathrm{eV}$ around the $\Gamma$-point. (b) Illustration of the real space electron density (magenta) reconstructed from the PMM shown in (a). The electron density cutoff is determined by a hierarchical cluster analysis ${ }^{35}$. The backdrop of the molecular lattice of the first layer is drawn as a guide to the eye.

total intensity of the molecular features. While on clean Ag substrate, the lifetime of electronic excitation is typically a few femtoseconds ${ }^{34}$, the observed dynamics around the $\Gamma$-point (circle B) is about two orders of magnitude slower. We thus suggest an interaction with silver sp-bands as reflected by the dynamical response of the overall shape in the PMMs of $\mathrm{LUMO}_{1 \mathrm{st}}$ and $\mathrm{HOMO}_{1 \mathrm{st}}$ and in particular of the intensity in around $\Gamma$-point. In contrast, the $\mathrm{HOMO}_{2 \mathrm{nd}}$ shows almost no change over time, indicating less interaction between second layer and substrate.

In Fig. 4 (a) we present a feature at $E_{B}=-400 \mathrm{meV}$, i.e. above Fermi level, that is temporarily visible from $\tau_{\text {Delay }}=400 \mathrm{fs}$ until $\sim 1200 \mathrm{fs}$. At first sight it resembles the intensity around the $\Gamma$-point belonging to silver sp-bands. For a pump-probe experiment on clean $\operatorname{Ag}(110)$, this is absent. Hence, we attribute the appearance of the signal in Fig. 4 (a) to the interaction of adsorbed pentacene with the silver substrate. By approximating the final state with a plane wave, we Fourier transform the PMM in Fig. 4 (a) to reconstruct the electron density in real space ${ }^{6}$. Since there are no apparent nodal planes of the intensity, we use a constant phase in the transformation and obtain an electron distribution expanding $13.8 \AA$ in [001] direction and $12.2 \AA$ in [-100] direction. The cutoff of the square of the wave function is determined by a hierarchical cluster analysis ${ }^{35}$. It suppresses artifical oscillations after the Fourier transformation of the measured PMM. In Fig. 4(b) we have projected the resulting square of the wave function onto a real space illustration of the first monolayer of a bilayer pentacene film on $\operatorname{Ag}(110)$. The size of the real space electron density extends to the neighboring molecule and differs from any unoccupied molecular orbital. This rather localized state above the Fermi level implies that the ordered molecules are essential for its formation. We thus conclude that the proximity of the first layer to the substrate and the molecule-substrate interaction is leading to this state.

In summary, we have demonstrated that time-resolved orbital tomography experiments for molecular thin film investigations are feasible at SASE FELs, and established the experimental conditions for sub-picosecond studies without interfering space charge induced effects and radiation damage. We confirmed an essential first step with the interpretation and identification of molecular orbitals in the time-resolved PMMs on an ultrashort time scale. Given the upcoming improvements in FEL time resolution towards sub-femtosecond and progress in the theoretical framework based on quantum electrodynamics ${ }^{36}$, time-resolved tomography of molecular wave functions during chemical reactions will provide unprecedented insight into photon-induced dynamics.

\section{Acknowledgement}

This work is dedicated to Prof. Dr. Wilfried Wurth, who passed away on May 8, 2019. We thank Andreas Oelsner for his technical assistance. The authors would like to thank FLASH for beamtime, and the staff of FLASH for their support during the experiment. We acknowledge financial support from the DFG through the Würzburg-Dresden Cluster of Excellence on Complexity and Topology in Quantum Matter - ct.qmat (EXC 2147, project-id 39085490), the excellence cluster "The Hamburg Centre for Ultrafast Imaging - Structure, Dynamics and Control of Matter at the Atomic Scale" of the Deutsche 
Forschungsgemeinschaft (DFG EXC 1074), the SFB 925 (project B2), and projects SCHO1260/4-2 and RE1469/12-2.

\section{Contributions}

M.S. designed the experiment and performed the data analysis. C.M. and K.B. performed data analysis. M.S., K.B., C.H.M., T.R.F.P., M.R., C.K., W.L., W.M.I., F.R., D.K., M.H., F.P. and W.W. conducted the experiment. M.S., K.B. and C.M. wrote the manuscript. C.H.M., T.R.F.P., C.K., W.M.I., F.R., D.K., M.H., F.P., A.S., S.M, W.W., and A.M. contributed to scientific discussions.

\section{References}

1. Dantus, M., Rosker, M. J. \& Zewail, A. H. Real-time femtosecond probing of "transition states" in chemical reactions. The J. Chem. Phys. 87, 2395-2397 (1987). URL http://aip.scitation.org/doi/10.1063/1.453122. DOI $10.1063 / 1.453122$.

2. Zewail, A. H. Laser Femtochemistry. Science 242, 1645-1653 (1988). URL http: / / science. sciencemag.org/ content/242/4886/1645. DOI 10.1126/science.242.4886.1645.

3. Petek, H., Weida, M. J., Nagano, H. \& Ogawa, S. Real-Time Observation of Adsorbate Atom Motion Above a Metal Surface. Science 288, 1402-1404 (2000). URL http: / / science. sciencemag.org/content/288/5470/1402. DOI 10.1126/science.288.5470.1402.

4. Frischkorn, C. \& Wolf, M. Femtochemistry at Metal Surfaces: Nonadiabatic Reaction Dynamics. Chem. Rev. 106, 4207-4233 (2006). URL https: / / doi.org/10.1021/cr050161r. DOI 10.1021/cr050161r.

5. Ziroff, J., Forster, F., Schöll, A., Puschnig, P. \& Reinert, F. Hybridization of Organic Molecular Orbitals with Substrate States at Interfaces: PTCDA on Silver. Phys. Rev. Lett. 104, 233004 (2010). URL https : / / i ink . aps . org/doi/ 10.1103/PhysRevLett.104.233004. DOI 10.1103/PhysRevLett.104.233004. Publisher: American Physical Society.

6. Puschnig, P. et al. Reconstruction of molecular orbital densities from photoemission data. Sci. (New York, N.Y.) 326, 702-706 (2009). DOI 10.1126/science.1176105.

7. Dauth, M. et al. Orbital Density Reconstruction for Molecules. Phys. Rev. Lett. 107, 193002 (2011). URL https : //link.aps.org/doi/10.1103/PhysRevLett.107.193002. DOI 10.1103/PhysRevLett.107.193002.

8. Weiß, S. et al. Exploring three-dimensional orbital imaging with energy-dependent photoemission tomography. Nat. Commun. 6, 8287 (2015). URL https: / /www . nature.com/articles/ncomms 9287. DOI 10.1038/ncomms9287.

9. Graus, M. et al. Electron-Vibration Coupling in Molecular Materials: Assignment of Vibronic Modes from Photoelectron Momentum Mapping. Phys. Rev. Lett. 116, 147601 (2016). URL https://link.aps.org/doi/10.1103/ PhysRevLett.116.147601. DOI 10.1103/PhysRevLett.116.147601.

10. Lüftner, D. et al. Understanding the photoemission distribution of strongly interacting two-dimensional overlayers. Phys. Rev. B 96, 125402 (2017). URL https: / / ink.aps.org/doi/10.1103/PhysRevB.96.125402. DOI 10.1103/PhysRevB.96.125402.

11. Grimm, M. et al. Molecular orbital imaging beyond the first monolayer: Insights into the pentacene/ $\operatorname{Ag}(110)$ interface. Phys. Rev. B 98, 195412 (2018). URL https://link.aps.org/doi/10.1103/PhysRevB.98.195412. DOI 10.1103/PhysRevB.98.195412.

12. Dauth, M. et al. Perpendicular Emission, Dichroism, and Energy Dependence in Angle-Resolved Photoemission: The Importance of The Final State. Phys. Rev. Lett. 117, 183001 (2016). URL https://Iink.aps.org/doi/10. 1103 /PhysRevLett.117.183001. DOI 10.1103/PhysRevLett.117.183001.

13. Metzger, C. et al. Plane-wave final state for photoemission from nonplanar molecules at a metal-organic interface. Phys. Rev. B 101, 165421 (2020). URL https://link.aps.org/doi/10.1103/PhysRevB.101.165421. DOI 10.1103/PhysRevB.101.165421. Publisher: American Physical Society.

14. Wießner, M. et al. Complete determination of molecular orbitals by measurement of phase symmetry and electron density. Nat. Commun. 5, 4156 (2014). URL https://www.nature.com/articles/ncomms5156. DOI $10.1038 /$ ncomms5156.

15. Lüftner, D. et al. Imaging the wave functions of adsorbed molecules. Proc. Natl. Acad. Sci. 111, 605 (2014). URL http://www.pnas.org/content/111/2/605. abstract. DOI 10.1073/pnas.1315716110. 
16. Kliuiev, P. et al. Algorithms and image formation in orbital tomography. Phys. Rev. B 98, 085426 (2018). URL https://link.aps.org/doi/10.1103/PhysRevB.98.085426. DOI 10.1103/PhysRevB.98.085426.

17. Heyl, C. M., Arnold, C. L., Couairon, A. \& L'Huillier, A. Introduction to macroscopic power scaling principles for high-order harmonic generation. J. Phys. B: At. Mol. Opt. Phys. 50, 013001 (2016). URL https: // doi .org/10. $1088 \div 2 \mathrm{~F} 1361-6455 \div 2 \mathrm{~F} 50 \div 2 \mathrm{~F} 1 \div 2 \mathrm{~F} 013001$. DOI 10.1088/1361-6455/50/1/013001.

18. Tiedtke, K. et al. The soft x-ray free-electron laser FLASH at DESY: beamlines, diagnostics and end-stations. New J. Phys. 11, 023029 (2009). URL https://doi.org/10.1088\%2F1367-2630\%2F11\%2F2\%2F023029. DOI $10.1088 / 1367-2630 / 11 / 2 / 023029$.

19. Dell'Angela, M. et al. Vacuum space charge effects in sub-picosecond soft X-ray photoemission on a molecular adsorbate layer. Struct. Dyn. 2, 025101 (2015). URL https://aca.scitation.org/doi/full/10.1063/1.4914892. DOI 10.1063/1.4914892.

20. Amy, F. et al. Radiation damage to alkyl chain monolayers on semiconductor substrates investigated by electron spectroscopy. The J. Phys. Chem. B 110, 21826-21832 (2006). DOI 10.1021/jp063614k.

21. Hellmann, S., Ott, T., Kipp, L. \& Rossnagel, K. Vacuum space-charge effects in nano-ARPES. Phys. Rev. B 85, 075109 (2012). URL https://link.aps.org/doi/10.1103/PhysRevB. 85.075109. DOI 10.1103/PhysRevB.85.075109.

22. Schönhense, G. et al. Correction of the deterministic part of space-charge interaction in momentum microscopy of charged particles. Ultramicroscopy 159, 488-496 (2015). URL https://linkinghub.elsevier.com/retrieve/ pii/s0304399115001266. DOI 10.1016/j.ultramic.2015.05.015.

23. Schönhense, B. et al. Multidimensional photoemission spectroscopy-the space-charge limit. New J. Phys. 20, 033004 (2018). URL https://doi.org/10.1088\%2F1367-2630\%2Faaa262. DOI 10.1088/1367-2630/aaa262.

24. Congreve, D. N. et al. External Quantum Efficiency Above $100 \%$ in a Singlet-Exciton-Fission-Based Organic Photovoltaic Cell. Science 340, 334 (2013). URL http://science. sciencemag.org/content/340/6130/334. abstract. DOI 10.1126/science.1232994.

25. Chan, W.-L. et al. Observing the Multiexciton State in Singlet Fission and Ensuing Ultrafast Multielectron Transfer. Science 334, 1541-1545 (2011). URL https: //science.sciencemag. org/content/334/6062/1541. DOI 10.1126/science. 1213986.

26. Martins, M. et al. Monochromator beamline for FLASH. Rev. Sci. Instruments 77, 115108 (2006). URL https: //aip.scitation.org/doi/abs/10.1063/1.2364148. DOI 10.1063/1.2364148.

27. Schönhense, G., Medjanik, K. \& Elmers, H.-J. Space-, time- and spin-resolved photoemission. J. Electron Spectrosc. Relat. Phenom. 200, 94-118 (2015). URL http: / www. sciencedirect.com/science/article/pii/ S0368204815001243. DOI 10.1016/j.elspec.2015.05.016.

28. Kutnyakhov, D. et al. Time- and momentum-resolved photoemission studies using time-of-flight momentum microscopy at a free-electron laser. Rev. Sci. Instruments 91, 013109 (2020). URL https://aip.scitation.org/doi/full/ $10.1063 / 1.5118777$. DOI 10.1063/1.5118777.

29. Liebsch, A. Theory of photoemission from localized adsorbate levels. Phys. Rev. B 13, 544-555 (1976). URL https : //link.aps.org/doi/10.1103/PhysRevB.13.544. DOI 10.1103/PhysRevB.13.544.

30. Fujikawa, T. \& Arai, H. Many-body theory of core-level photoemission by Keldysh Green's functions. J. Electron Spectrosc. Relat. Phenom. 123, 19-46 (2002). URL http://www. sciencedirect.com/science/article/ pii/S036820480100370x. DOI 10.1016/S0368-2048(01)00370-X.

31. Hasegawa, S. et al. Molecular orientation in thin films of bis(1,2,5-thiadiazolo)-p-quinobis(1,3-dithiole) on graphite studied by angle-resolved photoelectron spectroscopy. Phys. Rev. B 48, 2596-2600 (1993). URL https: / / link . aps . org/ doi/10.1103/PhysRevB.48.2596. DOI 10.1103/PhysRevB.48.2596.

32. Frisch, M. et al. Gaussian 03, revision c. 02; Gaussian. Inc., Wallingford, CT 4 (2004).

33. Xiang, B., Li, Y., Pham, C. H., Paesani, F. \& Xiong, W. Ultrafast direct electron transfer at organic semiconductor and metal interfaces. Sci. Adv. 3, e1701508 (2017). URL https://advances. sciencemag.org/content/3/11/ e1701508. DOI 10.1126/sciadv.1701508.

34. Bauer, M. \& Aeschlimann, M. Dynamics of excited electrons in metals, thin films and nanostructures. J. Electron Spectrosc. Relat. Phenom. 124, 225-243 (2002). URL http: / www. sciencedirect.com/science/article/ $\mathrm{pii/S} 0368204802000567$. DOI 10.1016/S0368-2048(02)00056-7. 
35. Arifin, A. Z. \& Asano, A. Image segmentation by histogram thresholding using hierarchical cluster analysis. Pattern Recognit. Lett. 27, 1515-1521 (2006). URL http://www.sciencedirect.com/science/article/pii/ S0167865506000699. DOI 10.1016/j.patrec.2006.02.022.

36. Popova-Gorelova, D. Imaging Electron Dynamics with Ultrashort Light Pulses: A Theory Perspective. Appl. Sci. 8, 318 (2018). URL https: / / www . mdpi.com/2076-3417/8/3/318. DOI 10.3390/app8030318. Number: 3 Publisher: Multidisciplinary Digital Publishing Institute. 


\section{Figures}

(a)

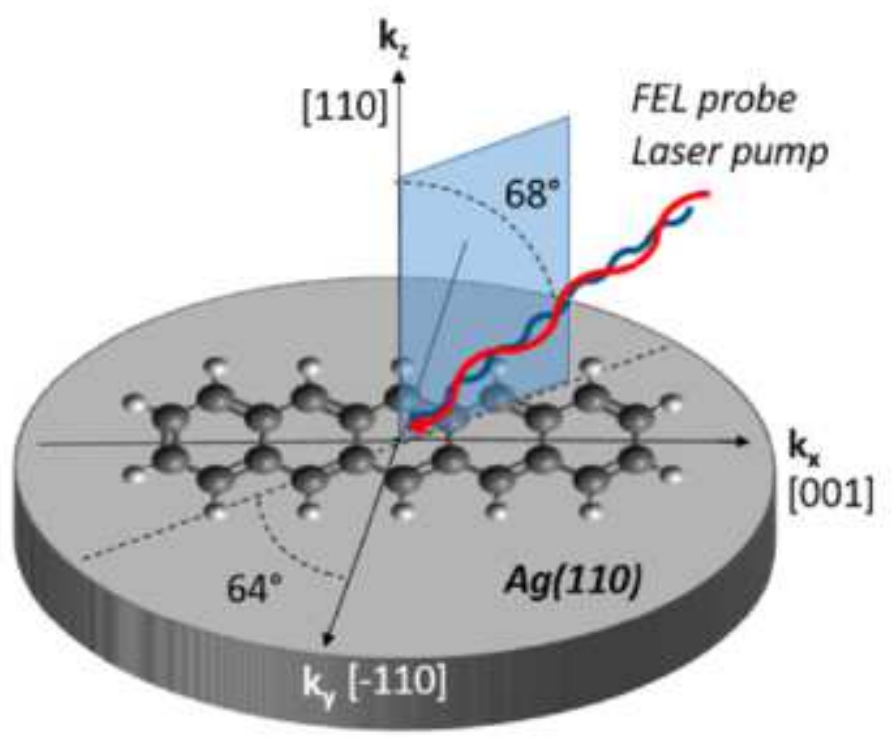

(b)

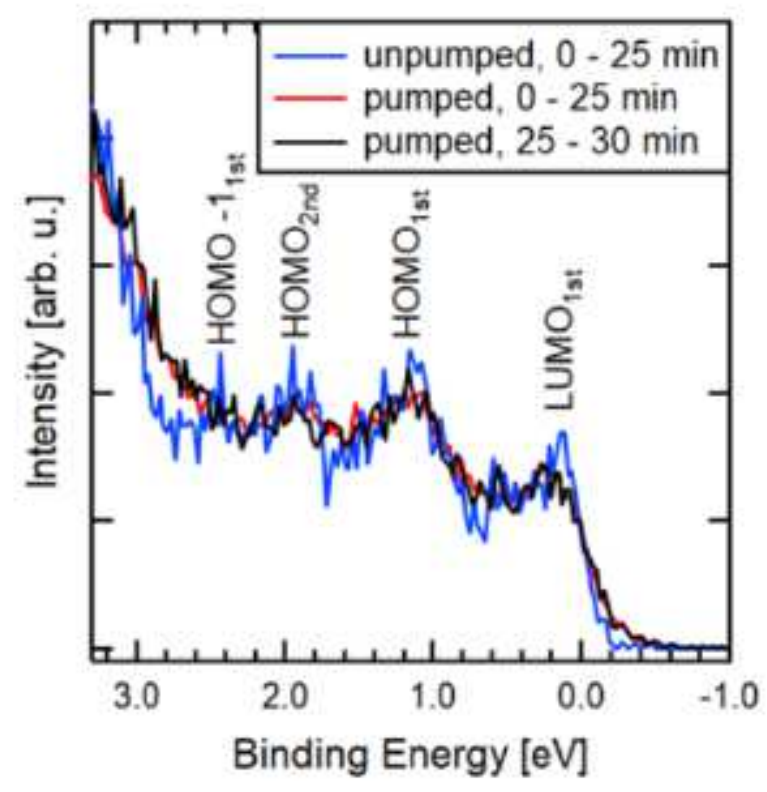

Figure 1

(a) Schematic illustration of the experimental geometry. All of the pentacene molecules are adsorbed with the long molecular axis along the [ll 001$]$ direction of the silver substrate. Pump and probe pulses are aligned to coincide in the same sample region. (b) Time-integrated photoelectron spectra for a bilayer of pentacene on $\mathrm{Ag}(110)$ with an excitation energy of $\mathrm{h} \nabla=35 \mathrm{eV}$. The measurements are taken at the same sample position for a total measurement time of $30 \mathrm{~min}$. Within a bunch train of 330 pulses with $1 \mu \mathrm{s}$ spacing at a repetition rate of $10 \mathrm{~Hz}, 299$ bunches are optically pumped (red and black lines). The blue line shows the time integrated signals of the 31 unpumped bunches. LUM01st, HOMO1st, HOMO-11st of the first layer and HOMO2nd of the second layer are indicated in the spectra. The spectra are normalized at $2.2 \mathrm{eV}$ binding energy. The LUM01st is partly filled due to charge transfer from the substrate. 


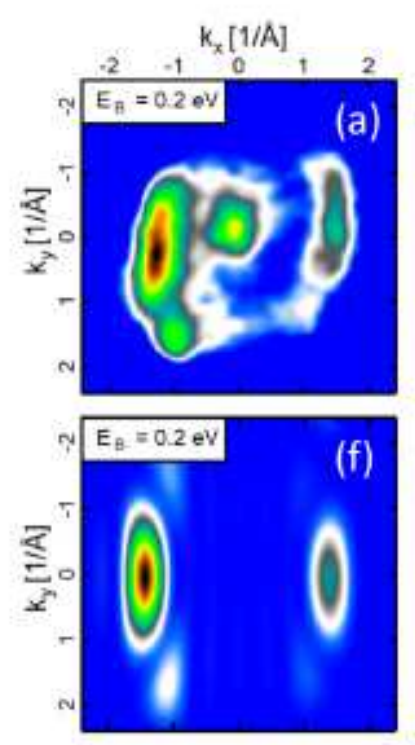

$\mathrm{LUMO}_{1 \mathrm{st}}$

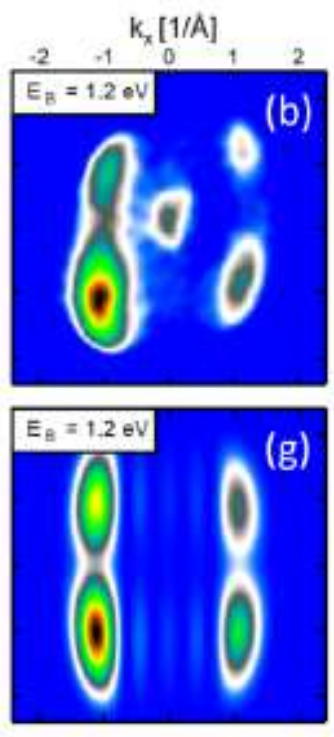

$\mathrm{HOMO}_{1 s t}$
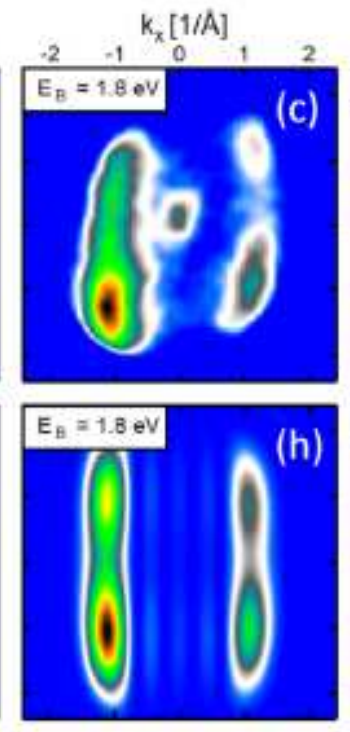

$\mathrm{HOMO}_{2 \text { nd }}$
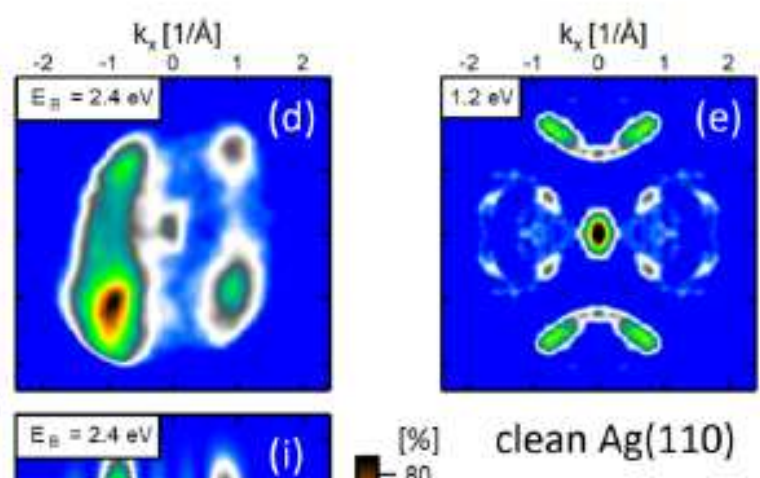

HOMO- $1_{15 t}$

Figure 2

Time-integrated measured $(a-d)$ and simulated $(f-i)$ photoelectron momentum maps (PMMs) for the pentacene valence orbitals at an excitation energy of $\mathrm{h} \nabla=35 \mathrm{eV}$ under simultaneous illumination with the pump and probe pulses. The partly filled LUMO1st (a), the HOMO1st (b), HOMO2nd (c), and the HOMO11 st (d) can be clearly distinguished from one another and identified by comparison to the simulations $(f-i)$. (e) An unpumped, symmetrized PMM of clean $A g(110)$ at $E B=1.2 \mathrm{eV}$ with otherwise identical parameters for comparison. The color scale has been adjusted to suppress the background signal. 
(a)
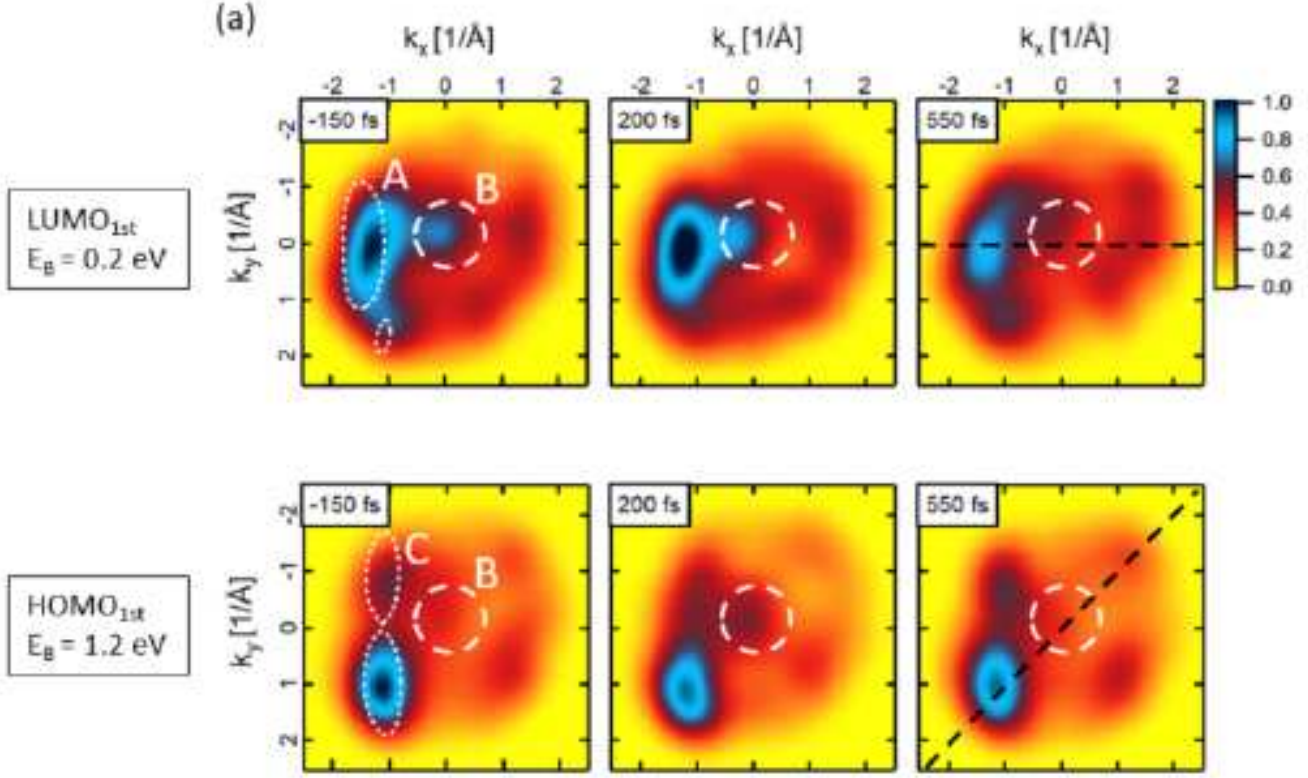

$\mathrm{HOMO}_{1 \mathrm{t}}$ $E_{\mathrm{B}}=1.2 \mathrm{eV}$

$\mathrm{HOMO}_{2 \text { nd }}$ $\mathrm{E}_{\mathrm{B}}=1.8 \mathrm{eV}$
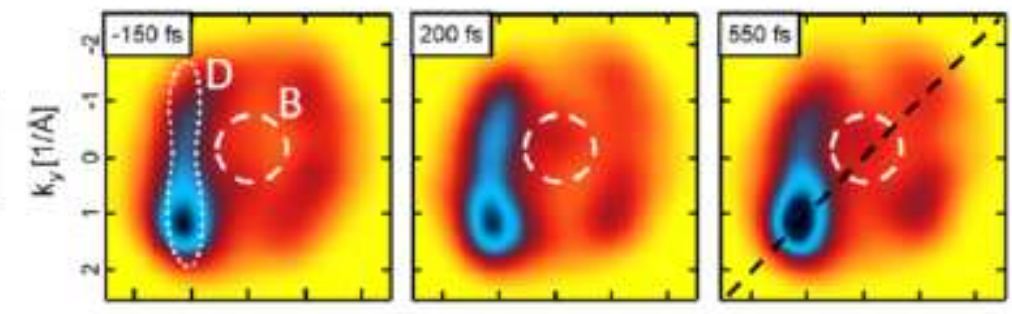

(b)
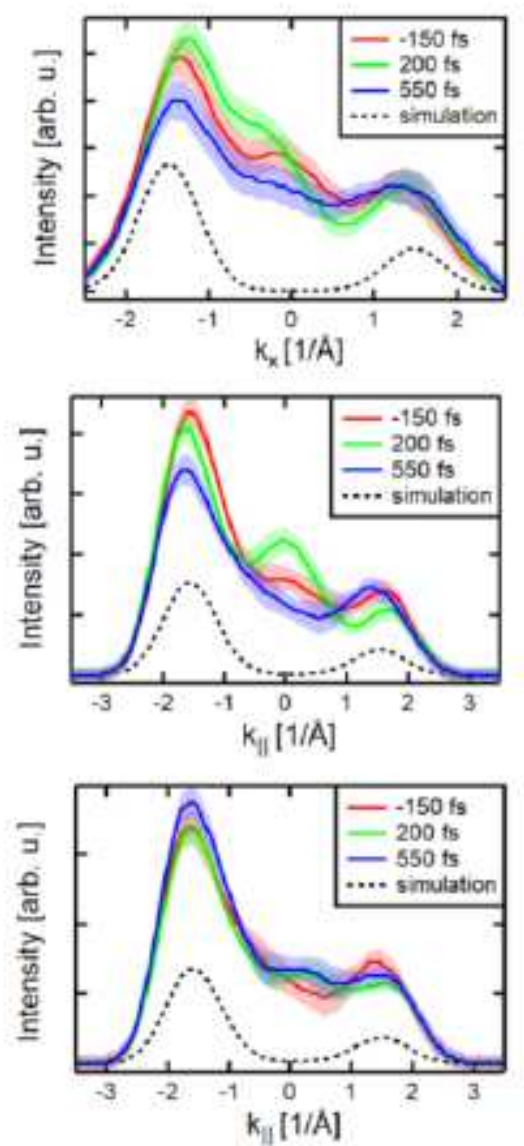

Figure 3

(a) Time evolution of photoelectron momentum maps (PMMs) of pentacene of the LUM01st (top row), $\mathrm{HOM01}$ st (middle row) and $\mathrm{HOMO} 2 \mathrm{nd}$ (bottom row) at $\mathrm{EB}=0.2 \mathrm{eV}, \mathrm{EB}=1.2 \mathrm{eV}$ and $\mathrm{EB}=1.8 \mathrm{eV}$ binding energy, respectively. The PMMs are integrated in intervals of $350 \mathrm{fs}$ for increasing pump-probe delays and smoothed in momentum space with a Gaussian filter. Contour lines A, C, D show the calculated PMMs (see Fig. $2(f-h)$ ) for the respective molecular orbitals. For clarity, the calculation is only shown on the left side of the PMM. The circle B indicates the intensity around the G-point. (b) Momentum distribution curves for LUM01st (top), HOMO1st (middle) and HOMO2nd (bottom) in the direction as indicated by the black dashed lines in the corresponding PMMs. The shaded bands reflect 1-s error-bars. 
(a)

(b)
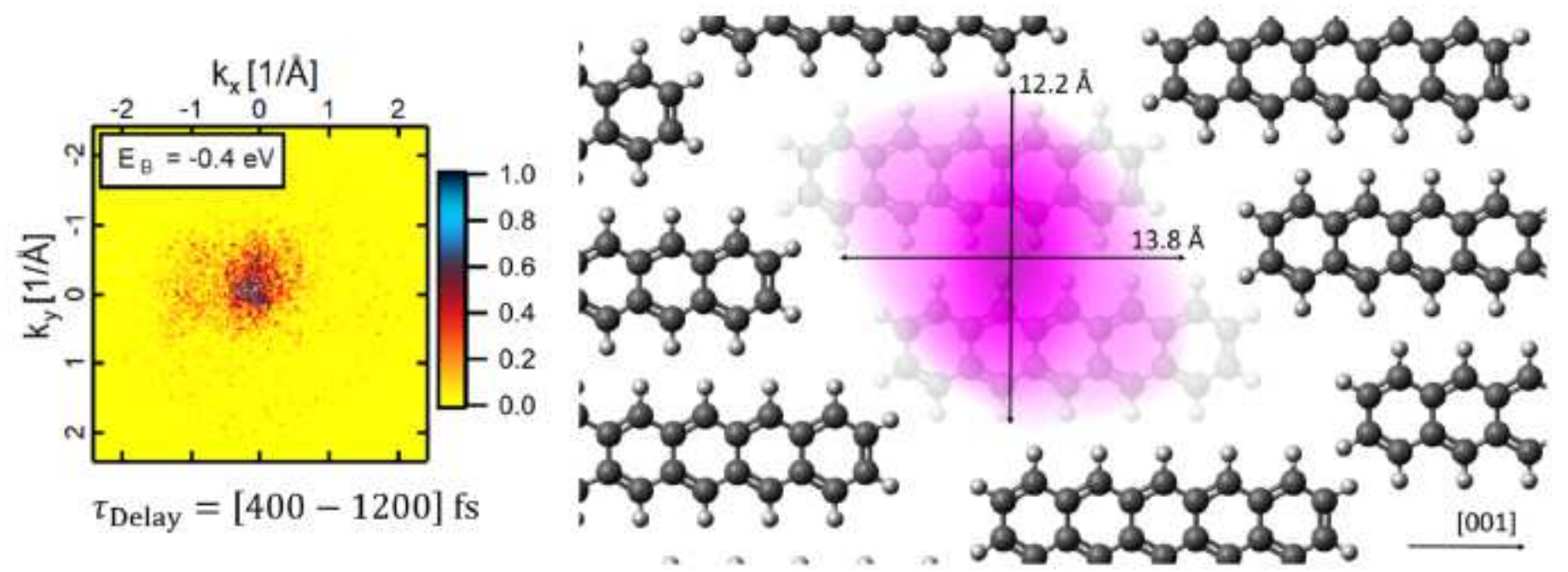

Figure 4

(a) Optical pumping enables the detection of an excited feature at EB $=-0.4 \mathrm{eV}$ around the G-point. (b) Illustration of the real space electron density (magenta) reconstructed from the PMM shown in (a). The electron density cutoff is determined by a hierarchical cluster analysis35. The backdrop of the molecular lattice of the first layer is drawn as a guide to the eye.

\section{Supplementary Files}

This is a list of supplementary files associated with this preprint. Click to download.

- supplementMS.pdf 